\title{
Fabry disease: a new challenge in endocrinology and metabolism?
}

\author{
U Feldt-Rasmussen, Å K Rasmussen, H Mersebach, K M Rosenberg ${ }^{1}$, L Hasholt $^{1}$ and S A Sorensen ${ }^{1}$ \\ Department of Medical Endocrinology, Rigshospitalet and ${ }^{1}$ Department of Medical Genetics, Institute of Medical Biochemistry and Genetics, University of \\ Copenhagen, Copenhagen, Denmark
}

(Correspondence should be addressed to U Feldt-Rasmussen, Department of Medical Endocrinology, Rigshospitalet, Blegdamsvej 9, DK-2100 Copenhagen, Denmark; Email: ufeldt@rh.dk)

Fabry disease ('angiokeratoma corporis diffusum') is a rare X-linked recessive metabolic glycosphingolipid storage disease caused by a deficiency of the lysosomal enzyme $\alpha$-galactosidase A ( $\alpha$-gal A). The estimated incidence is from 1 in 117000 to 1 in 40000 live births.

The glycosphingolipid substrate of $\alpha$-gal A is globotriaosylceramide $\left(\mathrm{Gb}_{3}\right)$, which accumulates at varying degrees within vulnerable cells, tissues and organs. Affected cell types include endothelial cells, pericytes, smooth muscle cells of the vascular system, renal epithelial cells, myocardial cells and dorsal ganglia neuronal cells. With increasing age of the patient, $\mathrm{Gb}_{3}$ progressively accumulates with a variety of manifestations from different organs.

Affected males, the Fabry hemizygotes, present with the most serious symptoms, but female carriers, the heterozygotes, may also show early onset and serious disease. The most dominant and frequent clinical features are angiokeratomas and acroparaesthesia with recurrent episodes of severe debilitating neuropathic pain in the extremities. The acroparaesthesias can cause what is known as 'Fabry crisis' with very strong and burning pain, particularly in the palms and under the feet, and associated with moderate fever and an elevated erythrocyte sedimentation rate. Fabry crises usually occur during exercise, fatigue or fast changes in temperature or humidity. Medications such as carbamazepine, phenytoin, gabapentin and lamotrigine may reduce the neuropathic pain.

Some patients experience loss of sensory or autonomic nerve functions resulting in decreased sweating, saliva and tear formation, as well as decreased intestinal motility and gastric paresis. Hypo- or anhydrosis with a decreasing possibility for heat dissipation may contribute to the elevation in body temperature seen during a crisis.

Accumulation of glycosphingolipids in the cornea gives rise to characteristic corneal opacities, visible by slit lamp. The lipids may also accumulate in the lens, the conjunctiva and the retina, usually without any visual disturbance. Visual impairment or blindness may be due to involvement of the optic nerve or retinal artery and is thus part of the vascular or neuropathic damage. Deafness is rare but many hemizygotes develop progressive hearing impairment. Furthermore, chronic airway obstruction has been demonstrated leading, although rarely, to pulmonary insufficiency.

Death usually occurs during the fourth or fifth decade of life from cardiovascular, cerebrovascular or renal disease. The increased mortality is due to microand macroangiopathy with accumulation of sphingolipids in the vascular system of the heart, kidneys and the brain. Cerebral strokes are common in Fabry patients as is progressive dementia due to ischaemia. Dilative arteriopathy of the vertebrobasilar circulation with resulting haemorrhagic strokes has also been described. Most patients have proteinuria and progressive renal failure may occur in the third, fourth or fifth decades. Cardiac hypertrophy is common as are arrhythmias, valvular insufficiency, cardiac conduction abnormalities and obstruction of coronary arteries leading to ischaemic heart disease and myocardial infarction.

Clinical onset of the disease typically occurs during childhood or adolescence with recurrent episodes of severe, debilitating neuropathic pain in the extremities. This neuropathy can occur as young as 5 years of age or even younger. Hypohidrosis also occurs at a young age, while the severe vascular complications show a slow progression, with a clinical debut of organ failures from the third, fourth or fifth decade.

The diagnosis of Fabry disease is in most cases initially made by clinical symptoms, and is associated with considerable problems due to the clinical variability of symptoms and the sparse incidence of the disease. The neuropathic pain, Fabry crisis, cardiovascular disease and angiokeratomas can all be mistaken for other diseases. A mutation analysis is imperative for exact diagnosis.

Recently, a breakthrough in the treatment of Fabry patients has been accomplished by successful production of $\alpha$-gal A by genetic engineering and thus development of a direct protein-substitution therapy. This replacement therapy has been approved by the European Union. Two randomised trials of enzymereplacement therapy for Fabry disease have been completed $(1,2)$ preceded by corresponding safety 
studies. Both studies found that enzyme-replacement therapy was well tolerated and likely to be effective, and the results were commented on in an Editorial (3). The enzyme preparation differed in the two studies: $\alpha$-gal A used by National Institutes of Health (NIH) investigators (1) was synthesised in human skin fibroblasts (Replagal; Transkaryotic Therapies, Inc.), and that of the Mount Sinai Study Group (MSSG) (2) in Chinese hamster ovary cells (Fabrazyme; Genzyme). Also the dose per infusion differed, $0.2 \mathrm{mg} / \mathrm{kg}$ against $1.0 \mathrm{mg} / \mathrm{kg}$, resulting in infusion times of 40 and $240 \mathrm{~min}$ respectively. However, whether potency or other differences in the enzymes used affected the therapeutic effect is not certain, due to differences in selection of patients and endpoints. Both studies included only adult hemizygous men, 26 males and 56 males respectively. The benefit of the treatment in heterozygous (carrier) females and children is being studied in ongoing trials.

The NIH study (1) found a decrease in mesangial widening in the glomeruli of the kidney in the group of patients receiving $\alpha$-gal A and the MSSG study (4) a decrease in $\mathrm{Gb}_{3}$ concentration in liver and kidney biopsies of patients treated with $\alpha$-gal A compared with placebo groups. Both studies reported significant decreases in urine-sediment and plasma $\mathrm{Gb}_{3}$ concentrations. The NIH study (1) also found a stable glomerular filtration rate in the treated arm significantly different from that of the placebo group, which followed the expected decline of glomerular function in untreated patients. The NIH study (1) proved a decline in neuropathic score in Fabry patients treated with $\alpha$-gal A. The patients in the MSSG trial (2) were not selected for pain symptoms and remained on analgesics throughout the study and thus no significant improvement in pain between treated and placebo groups could be demonstrated. Furthermore, a significantly improved cardiac conduction was seen in the treated patients. Resting regional cerebral blood flow, measured by positron emission tomography, was significantly reduced in the treated group $(n=12)$ compared with the control group $(n=12)(5)$. A possible relationship between increased risk of stroke in Fabry disease and elevated cerebral blood flow is, however, unclear.

The patients included in the randomised clinical trials have in all instances been adult homozygotes with symptomatic disease. The results of these shortterm clinical trials have been promising in terms of both reduction of storage and clinical symptoms. However, end-stage kidney disease is most probably irreversible, which is also likely to be the case for advanced storage in other organs such as the heart, cerebrum, vessels etc. Presumably organ failure might be prevented by earlier onset of treatment. Thus, studies of patients with few symptoms - or none - are needed to demonstrate if early intervention is beneficial against development of organ manifestations and premature mortality.

A key issue is also the reimbursement policy of each country after formal approval of the therapy. It is an expensive treatment, in the order of more than $€ 100000$ per treated person per year. The patients need i.v. infusion of the enzyme at regular intervals (every second or each week), and therefore asymptomatic patients may renounce treatment. This raises the difficult questions of the selection of whom to treat, including at what age the treatment should be started, and whether symptoms of organ failure should be present or not before onset of therapy.

Causal active treatment of patients with Fabry disease has entered a new era of specific monitoring of organ involvements. It will be important even to monitor incipient signs of any organ failure in affected individuals in order to determine the treatment effect and observe possible progression. The diffuse organ manifestations of the disease are very similar to multiorgan involvements in other more frequent metabolic disorders such as diabetes mellitus and pituitary diseases. Thus medical endocrinology might be an appropriate speciality to manage and coordinate the treatment in collaboration with other organ specialists and geneticists. It is appropriate that these patients are treated by a specialist who is familiar with, interested in and capable of proper clinical care of this multispeciality disease and its treatment aspects (6).

\section{References}

1 Schiffman R, Kopp JB, Austin HA, Sabnis S, Moore DF, Weibel T et al. Enzyme replacement therapy in Fabry disease. A randomised controlled trial. Journal of the American Medical Association 2001 $2852743-2749$.

2 Eng C, Guffon N, Wilcox WR, Germain DP, Lee P, Waldek S et al. Safety and efficacy of recombinant human $\alpha$-galactosidase A replacement therapy in Fabry's disease. New England Journal of Medicine 2001345 9-16.

3 Pastores MP \& Ravi T. Enzyme replacement therapy for AndersonFabry disease. Lancet 2001358 601-603.

4 Eng CM, Banikazemi M, Gordon RE, Goldman M, Phelps R, Kim L et al. A phase 1/2 clinical trial of enzyme replacement in Fabry disease: pharmacokinetic, substrate clearance and safety studies. American Journal of Human Genetics 200168 711-722.

5 Moore DF, Scott LTC, Gladwin MT, Altarescu G, Kaneski C, Suzuki K et al. Regional cerebral hyperperfusion and nitric oxide pathway dysregulation in Fabry disease. Reversal by enzyme replacement therapy. Circulation $2001 \mathbf{1 0 4} 1506-1512$.

6 Peters FP, Sommer A, Vermeulen A, Cheriex EC \& Kho TL. Fabry's disease: a multidisciplinary disorder. Postgraduate Medical Journal $199773710-712$.

Received 8 March 2002

Accepted 14 March 2002 